\title{
Introducing a New NIST Reference Material: Multiwall Carbon Nanotube Soot
}

\author{
Ann N. Chiaramonti, Ryan M. White, Jason Holm, and Elisabeth Mansfield
}

Material Measurement Laboratory, National Institute of Standards and Technology, Boulder USA.

Multiwall carbon nanotubes (MWCNTs) play a significant role in the nascent nanotechnology industry, due to their remarkable combination of mechanical, thermal, and electrical properties. This material is used as a specialty additive, reinforcing a diverse group of composites, for sporting goods, automobile body panels, and anti-static coatings. More than 25 commercial products incorporate MWCNTs, and production volumes exceeded 500 tons per year in 2011. These volumes are expected to increase dramatically in the near future with 3000 tons/year predicted in 2015 due to growing applications in electronics, data storage, defense, energy, aerospace, and the automotive industry $[1,2]$. These new applications dictate higher performance and reliability and require tight control over the physical and chemical characteristics of the MWCNTs.

More than 80 companies worldwide produce nanotubes, more than two-thirds of which offer MWCNTs. Most MWCNTs are synthesized using pilot- or laboratory-scale processes. However, several companies have recently established large-scale production facilities, which lower costs and ensure better material uniformity [3]. Ensuring the quality of MWCNTs remains a challenge, especially given that different applications demand different degrees of purity - defined by bulk chemistry, surface chemistry, and size specificity. With the global MWCNT market poised to exceed \$1.9B in annual revenues in 2015, the need for improved quality control is paramount [4].

In direct response to the needs of industry and research institutions for a well-characterized multiwall carbon nanotube reference, we have recently created a new NIST Reference Material, RM8284 Multiwall Carbon Nanotube Soot. The source stock for RM8284 is a black granular commercial material primarily composed of MWCNT, amorphous carbon impurities, and residual cobalt/aluminum catalyst. Characterization of the RM includes thermogravimetric analysis, transmission electron microscopy (TEM), scanning electron microscopy (SEM), inductively coupled plasma mass spectroscopy and neutron activation analysis (including prompt gamma activation analysis and instrumental neutron activation analysis).

Here we will report on the results of our certification of RM8284 Multiwall Carbon Nanotube Soot, including measurement of number of walls (Figure 1), length (Figure 2), inner and outer diameter, ash content (catalyst), and purity. There will be a particular focus on the microscopy and microanalysisrelated metrology techniques used for length measurement, wall counting, and chemistry and an indepth discussion of uncertainty for each measurement method.

\section{References:}

[1] J. H. Lehman, M. Terrones, E. Mansfield, K. E. Hurst, V.Meunier. "Evaluating the characteristics of multiwall carbon nanotubes" Carbon, 49, 2581-2602, 2011.

[2] http://www.nanotechproject.org/inventories/consumer/

[3] "Bayer bets big on carbon nanotube production." Plastics Today (Jan 29, 2010).

[4] "The Global Market for Nanotubes to 2015: A realistic assessment", Research and Markets, December 2009. 
[5] This work is a contribution of NIST, an agency of the United States government. As such, it is not subject to copyright in the United States.
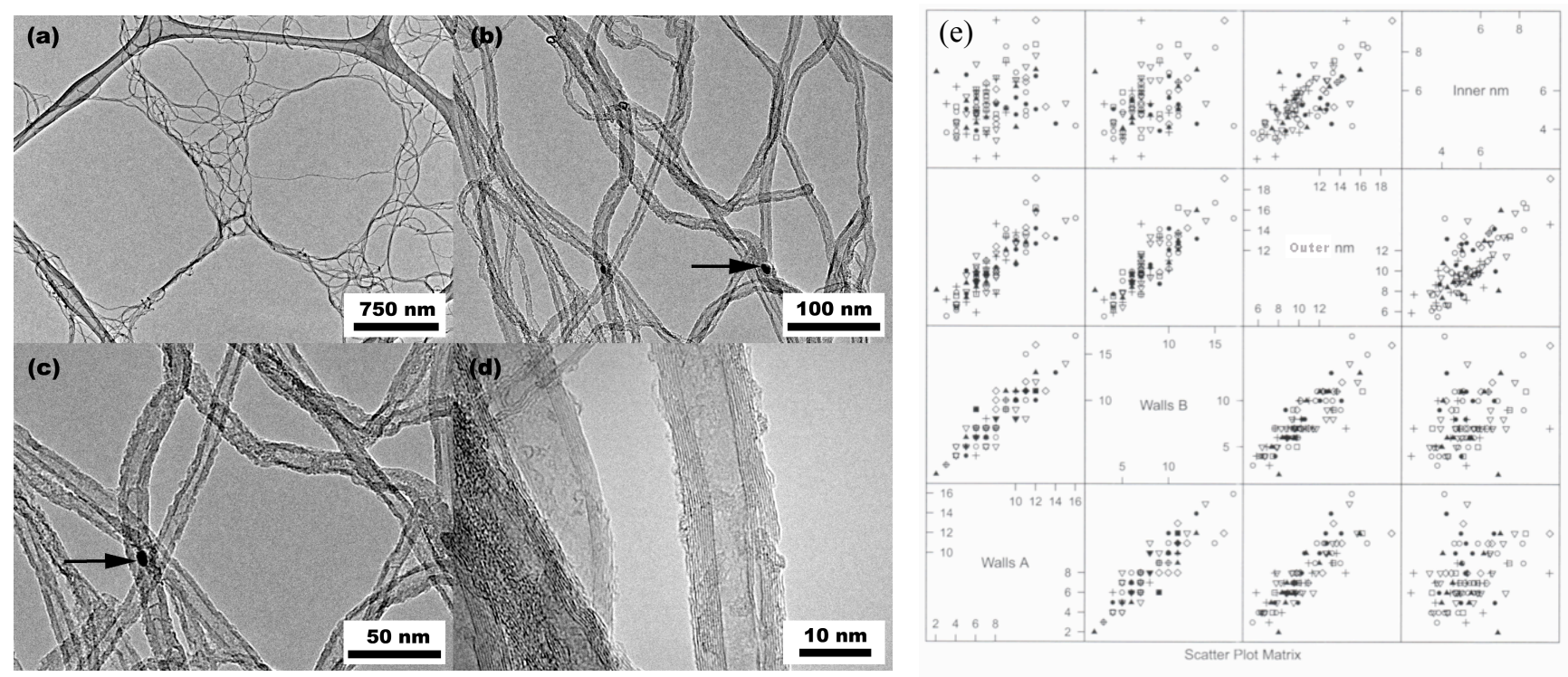

Figure 1. (a-d) TEM images of NIST RM8284 dispersed on a lacey carbon support grid. Residual catalyst can be seen (arrows) in images b and c. (e) Correlation matrix for measurement of number of walls on either side (A, B), inner diameter, and outer diameter.
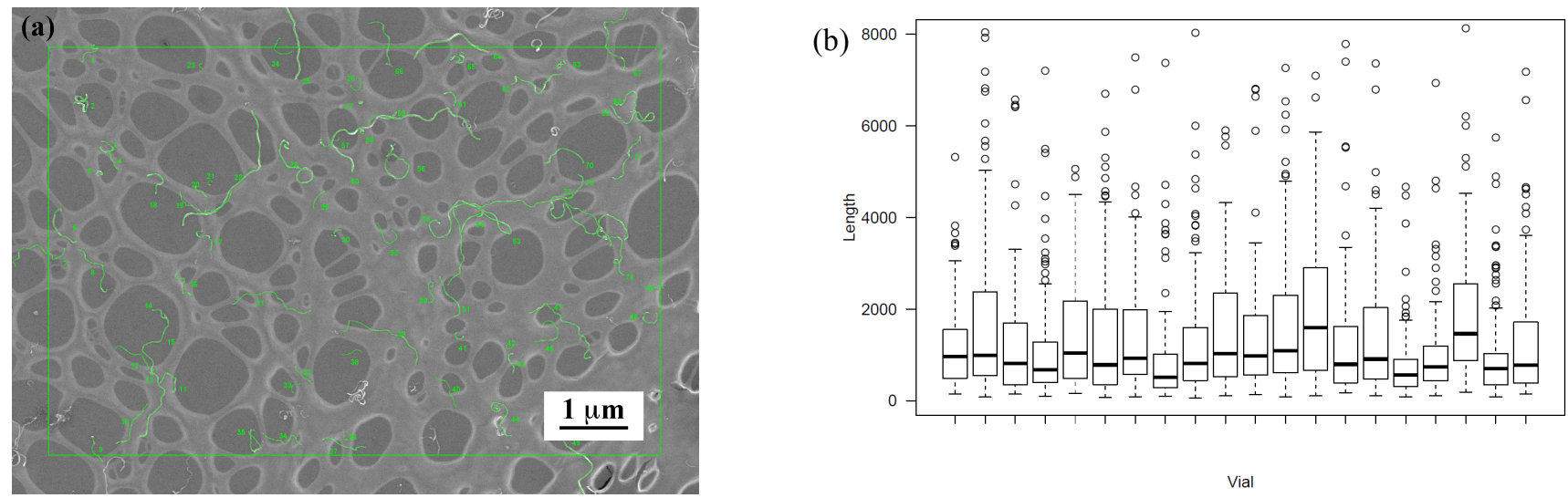

Figure 2. (a) Typical SEM image used for length measurement. (b) Results of the length measurements. Each box represents length measurements $25 \%$ to $75 \%$ of the data recorded for a specific sample. Individual outliers are represented with open circles. The dark line is the mean for each vial. Dotted brackets represent uncertainty in the measurement. Units of length are nanometers. 